\title{
Strong Enhancement of Light Extraction Efficiency in GaInAsP 2-D-Arranged Microcolumns
}

\author{
Toshihiko Baba, Member, IEEE, Kyoji Inoshita, Hiroko Tanaka, Jun Yonekura, Maiko Ariga, Akihiro Matsutani, \\ Tomoyuki Miyamoto, Fumio Koyama, Member, IEEE, and Kenichi Iga, Fellow, IEEE, Fellow, OSA
}

\begin{abstract}
This paper experimentally demonstrates the strong enhancement of light extraction efficiency in two-dimensionally arranged microcolumns. They were designed like a honeycomb photonic crystal and fabricated into GaInAsP-InP wafers by using the inductively coupled plasma etching. For the laterally directed light passing through the microcolumns, peculiar transmission characteristics were observed, which could be explained by the Bragg reflection theory, namely, the photonic bandgap (PBG). The measurement of spontaneous lifetime showed that the internal efficiency in the microcolumns was reduced by the surface recombination at sidewalls. In contrast, the light extraction efficiency evaluated from the measured photoluminescence intensity, and the internal efficiency was more than ten times that for a planar wafer. This was thought to be due to the expanded escape cone of internal light by the low effective refractive index, and also due to the strong diffraction and scattering of laterally directed light, which corresponds to the second-order Bragg condition. Such effects are expected not only in photonic crystals but also in some disordered structures. We expect this structure to allow a high-efficiency light-emitting diode (LED), since electronic elements needed for current injection devices can be added independently of the effects.
\end{abstract}

Index Terms-Extraction efficiency, GaInAsP-InP, microcavity light-emitting diode (LED), phase-resolved spectroscopy, photonic crystal, spontaneous lifetime, surface recombination.

\section{INTRODUCTION}

$\mathbf{P}$ HOTONIC crystals made of III-V compound semiconductors are expected to provide a novel performance in light emitters [1]. One interesting issue is the enhancement of light extraction efficiency in light-emitting diodes (LED's), which is normally less than $3 \%$ due to the narrow escape cone of internal light from a high-refractive-index semiconductor to the air.

Manuscript received August 13, 1999. This work was supported in part by the Ministry of Education, Science, Sports, and Culture under Grant-in-Aid 10210203

T. Baba, K. Inoshita, and M. Ariga are with the Division of Electrical and Computer Engineering, Yokohama National University, Yokohama 240-8501 Japan.

H. Tanaka was with the Division of Electrical and Computer Engineering, Yokohama National University, Yokohama 240-8501 Japan. She is now with NEC Corporation, Tokyo, Japan.

J. Yonekura was with the Division of Electrical and Computer Engineering, Yokohama National University, Yokohama 240-8501 Japan. He is now with Canon, Inc., Kawasaki, Japan.

A. Matsutani, T. Miyamoto, F. Koyama, and K. Iga are with the Precision and Intelligence Laboratory, Tokyo Institute of Technology, Yokohama 2268503 Japan.

Publisher Item Identifier S 0733-8724(99)08812-X.
The basic concept can be seen in a simple slab. The lengthened spontaneous lifetime has been observed for a thin GaAs-AlGaAs slab with low-refractive-index surroundings [2]. It simply demonstrated the theoretical expectation that the spontaneous emission can be controlled by the vacuum field fluctuation, namely, characteristics of optical modes [3]-[5]. A developed form of this concept is spontaneous emission control in a planar microcavity. It consists of a slab active layer sandwiched by a pair of distributed Bragg reflectors (DBR's). Some amount of spontaneous emission suppressed by the stopband of DBR's is transferred to the resonant mode emission efficiently extracted toward vertical directions [6]-[8]. An extraction efficiency of more than 20\% was theoretically expected for a typical GaAs-AlAs DBR [9] and experimentally observed in a GaInAs LED with the DBR's [10]. These values are limited by the existence of nonextracted guided and leaky modes. Further enhancement of the efficiency is expected for two-dimensional (2-D) photonic crystals. A high efficiency over $30 \%$ was theoretically estimated from one side of a semiconductor slab with air holes [11]. It owes to a photonic bandgap (PBG) that suppresses the guided mode emission and enhances the coupling of emission into air modes. Until now, this high efficiency has not been experimentally demonstrated. An expected problem is the surface recombination at sidewalls of holes, which degrades the internal efficiency. Another problem is its geometry, which makes a current injection device difficult. The top contact layer and metal electrodes necessary for injection devices will essentially change the expected effects, since the effects must strongly depend on upper and lower boundary conditions.

The photon recycling and the textured surface have been studied as other approaches. The photon recycling improves the extraction efficiency by the reabsorption of the nonextracted light [12]. It needs a high-reflectivity mirror to feed back the light to the active region. By this effect, over $70 \%$ extraction efficiency of the photoluminescence (PL) was achieved for a $\mathrm{GaAs}-\mathrm{AlGaAs}$ thin slab with a $\mathrm{SiO}_{2}-\mathrm{Au}$ mirror [13]. Also for this structure, the problem is the difficulty of making an injection device. In a planar microcavity LED, the improvement by this effect is small due to the inefficient reabsorption. Twice, an increase of the efficiency was observed in a GaInAsP-InP thin slab LED with an AuZn film used as an electrode and a feedback mirror [14]. This small increase was theoretically expected, since the reflectivity of the metal film was as low as $\sim 75 \%$ after the annealing process for making 
the ohmic contact. The effect of the textured surface also depends on the mirror reflectivity [15]. This method achieved $\sim 30 \%$ efficiency in an LED with a nonannealed metal mirror separated from electrodes.

This paper discusses the extraction efficiency in 2-Darranged semiconductor microcolumns, designed like a 2-D photonic crystal. We can suppose three effects for the enhancement of the efficiency. The first one is the expanded escape cone of the internal light by a low effective refractive index of the diluted semiconductor, namely, the average medium having a small filling factor of semiconductor columns. However, the laterally directed light cannot be extracted from a simple diluted semiconductor. The second effect is that the lateral light is converted to the light toward vertical directions by the diffraction in the air space between columns and the strong scattering at semiconductorair interfaces. The third effect is that the lateral emission is essentially inhibited by a PBG of columns as a photonic crystal. This results in the change of spontaneous lifetime. These three effects are similar to those discussed in [11]. Compared with a slab with air holes, microcolumns are advantageous on the following two points. The surface recombination will be smaller in columns, since the sidewall area exposed to the air is $1 / 3-1 / 5$ of that in air holes when assuming a constant filling factor of semiconductor [1]. The fabrication of an injection device will be much easier for columns, since a contact layer and metal electrodes can be added almost independently of the effects. We do not expect the photon recycling, so additional mirrors are not necessary.

In this study, we fabricated microcolumns and evaluated the PL intensity as well as the internal efficiency. As a result, a high extraction efficiency was evaluated, which was thought to be primarily due to the first two effects mentioned above. Distinctive features of this study are as follows.

1) GaInAsP-InP materials were used to suppress the surface recombination at sidewalls of the active layer as small as possible.

2) A honeycomb arrangement of columns was used, which was expected to exhibit the polarization-insensitive PBG [16] and minimize the surface recombination [1].

3) To obtain fine columns micrometers to submicrometers in diameter and several micrometers in height, the inductively coupled plasma (ICP) etching was used, which was known as a high-speed etching method of silicon for micromachine elements, etc.

4) The PBG was evaluated by using the direct optical pump of columns and detection of the PL. This simplifies the optical alignment and reduces the influence of the diffraction and absorption.

5) To evaluate separately the extraction efficiency and the internal efficiency, the spontaneous lifetime was measured. A phase-resolved spectroscopy was used, which allowed the measurement at a wavelength $\lambda>$ $1.1 \mu \mathrm{m}$.

We describe the fabrication of microcolumns in Section II, the observation of the PBG in Section III, the measurement of the spontaneous lifetime and the evaluation of the internal efficiency in Section IV, and the measurement of the PL intensity and the evaluation of the extraction efficiency in Section V. Last, we discuss the origin of the high efficiency and the applicability to an injection device.

\section{FABRICATION}

We prepared two epitaxial wafers $\mathrm{A}$ and $\mathrm{B}$ grown on InP substrates by a metal organic chemical vapor deposition. Wafer A included 19 GaInAs lattice-matched quantum wells (QW's) and an InP upper cladding. They were all undoped. The thickness of QW's widely ranged from 3 to $20 \mathrm{~nm}$ so that it exhibited a broad emission spectrum from $\lambda=$ 1.2 to $1.65 \mu \mathrm{m}$. We used this wafer to evaluate the PBG. Wafer B was a typical laser diode wafer including eight GaInAsP compressively strained QW's of $\sim 4 \mathrm{~nm}$ in each thickness, GaInAsP strain-compensated barriers, a GaInAsP gradient-index separate confinement heterostructure, and upper p- and lower n-InP claddings. The peak emission wavelength was $1.52 \mu \mathrm{m}$ at room temperature. We used this wafer to evaluate the extraction efficiency.

To observe the dependence of the PBG frequency and the light extraction efficiency on the structural design, we prepared various diameter $2 r$ of columns and pitch $a$ of the honeycomb arrangement $(2 r=0.3-2.0 \mu \mathrm{m}$ and $a=1.15-7.75 \mu \mathrm{m})$. They were not designed independently, but designed as they satisfied some filling factor $f$ of columns. The filling factor $f$ targeted was 0.12 . It gives the polarization insensitive PBG at $\lambda \sim 1.5 \mu \mathrm{m}$, when the refractive index of columns is 3.38 and $a=1.15-1.35 \mu \mathrm{m}$ [16]. We prepared patterns with various $f$ up to 0.17 , i.e., larger diameters of columns than the optimum one, to compensate the unwanted reduction of the diameter in the fabrication process

The fabrication process was as follows. First, Ti was evaporated on the wafers. Second, the honeycomb pattern of circular holes was drawn on a positive resist using the electron beam lithography. After $\mathrm{Cr}$ was evaporated, the pattern was reversed to that of $\mathrm{Cr}$ dots by the liftoff technique. The reversed pattern was transferred to $\mathrm{Ti}$ using the $\mathrm{CF}_{4}$ reactive ion etching. Finally, the wafer was etched by the ICP etching with $\mathrm{Cl}_{2}$ and Xe gases. The detail of the ICP etching has been reported previously [17]. Fig. 1 shows scanning electron micrographs (SEM's) of formed columns. The average roughness at sidewalls estimated was 1-nm order. The height and maximum aspect ratio were $2.5 \mu \mathrm{m}$ and 8.3 , respectively. The sidewall of upper $1.2 \mu \mathrm{m}$ was almost vertical, while that of lower 1.3 $\mu \mathrm{m}$ was tilted by the microloading effect. The variation of the top diameter $2 r$ was $15 \%$ in rms. The scattering matrix analysis indicated that this variation in this type of photonic crystal weakens the PBG effect by half [18].

\section{OBSERVATION OF PBG}

For the observation of the PBG, transmission spectra were measured. We used wafer A as a light source with the wide spectral range. This simple source is advantageous, since the PL gives a high-power density as a white source. The PL from the wafer was detected from a lateral direction by a sharpened single-mode fiber with a tip radius of $\sim 1 \mu \mathrm{m}$ and analyzed 


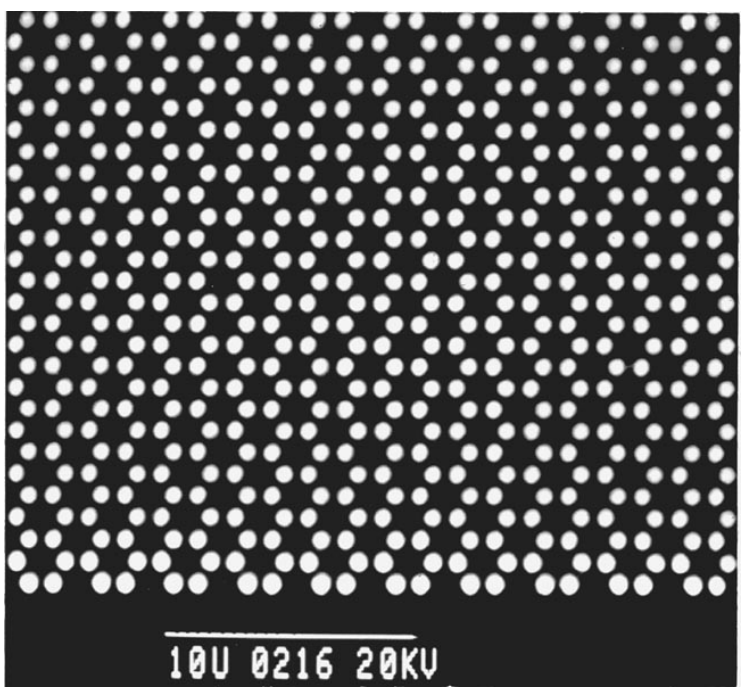

(a)

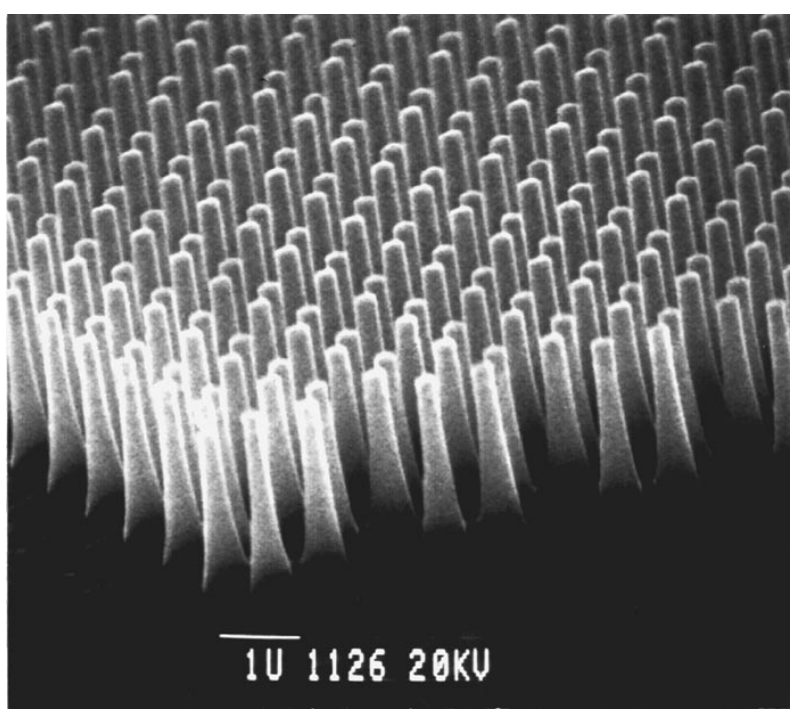

(b)

Fig. 1. SEM views of formed photonic crystal: (a) top view and (b) side view.

by an optical spectrum analyzer. All the measurements were carried out at room temperature.

Four measurement setups, as illustrated in Fig. 2, were tested. The setups shown in Fig. 2(b) and (c) were advantageous regarding the easy optical alignment. However, a long distance from the emitter to the fiber tip in Fig. 2(b) reduced the contrast of the transmitted light passing through columns against the direct light from the emitter to the fiber. It also caused the reabsorption of light in columns. But it was difficult to reduce the distance up to several micrometers by the cleavage of the wafer. In Fig. 2(c), the detection of the direct light was excluded by a long waveguide, but the reabsorption was enhanced and transmission spectra were seriously changed. The reabsorption could be excluded by the transparent columns in Fig. 2(d), but the optical alignment was the most difficult and unstable. Thus, we finally used the direct pump of columns Fig. 2(a). In this setup, the optical alignment

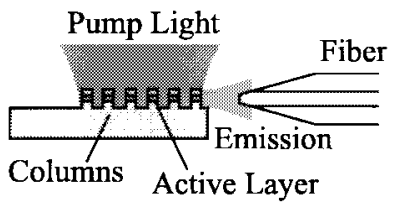

(a)

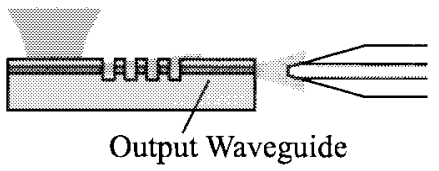

(c)

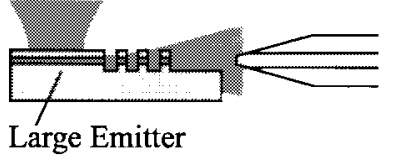

(b)

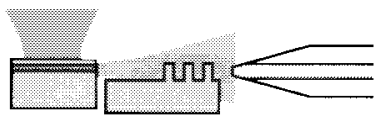

(d)
Fig. 2. Measurement setups for PBG: (a) direct optical pump of columns, (b) monolithic integration of large emitter and columns, (c) additional integration of output waveguide [19], and (d) hybrid integration of wafer A and another InP wafer with columns [20].

was easy, and the reabsorption was excluded by a sufficient pump, as described below.

In the measurement, the wafer was cleaved into two pieces at the center of the photonic crystal of $60 \times 150 \mu \mathrm{m}^{2}$ in total area. First, the center of the crystal on one of the cleaved wafers was pumped by continuous-wave (CW) light of $\lambda$ $=840 \mathrm{~nm}$. The focused spot diameter of the pump light was $\sim 30 \mu \mathrm{m}$, and the irradiated power density was $\sim 30 \mathrm{~kW} / \mathrm{cm}^{2}$. The fiber tip was put close to the cleaved facet. When the fiber tip was moved up and down along the vertical direction, a position was found, at which the PL intensity at $\lambda<1.3 \mu \mathrm{m}$ was drastically weakened to less than $-10 \mathrm{~dB}$ of that before. We thought that it was the closest position of the fiber tip to the columns at the wafer edge, since the spectral change should be caused by the reabsorption of the PL in columns between the pumped area and the wafer edge. The fiber tip was fixed at this position, and the pumped area was moved close to the fiber tip. Almost perfect recovery of the intensity at $\lambda<1.3 \mu \mathrm{m}$ was observed. This means the extinction of the reabsorption. Under this condition, transmission spectra were evaluated.

To observe the dependence of the PBG on the structural design, we selected four samples with different pitch $a$ and a constant filling factor $f=0.12$. The PL was detected from the $\Gamma-X$ direction in the Brillouin zone of the photonic crystal. Fig. 3 shows transmission spectra given by the ratio of observed spectra to a reference spectrum, which was obtained by averaging spectra for a simple wafer without columns. For each sample, a decrease of $2-3 \mathrm{~dB}$ in transmission was observed in each spectral range. This spectral range red-shifted with the increase of the pitch $a$ and diameter $2 r$. Fig. 3 compares experimental results with simulated ones obtained by the scattering matrix method. In this simulation, the model was 2-D, i.e., the infinite height of transparent columns and the uniform field distribution along the columns were assumed. In addition, all columns were assumed as light sources emitting cylindrical waves. Therefore, due to the direct detection of light from edge columns, the decrease of light at the PBG is limited to as little as $\sim 6 \mathrm{~dB}$ in the simulation. The PBG as wide as $\sim 300 \mathrm{~nm}$ is due to the observation of light from one direction assumed in the simulation. As seen in Fig. 3, experimentally observed spectra roughly accorded with 


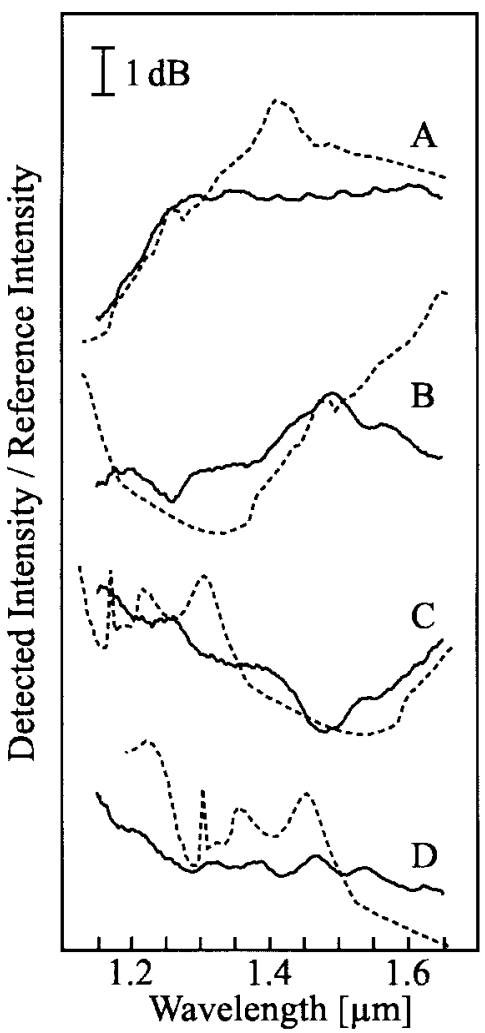

Fig. 3. Transmission spectra evaluated for four crystals A-D. Solid and dotted curves indicate experimental and simulated results, respectively. Pitch $a=1.15 \mu \mathrm{m}$ and diameter $2 r=0.30 \mu \mathrm{m}$ for A, 1.35 and $0.35 \mu \mathrm{m}$ for B, 1.55 and $0.40 \mu \mathrm{m}$ for $\mathrm{C}$, and 1.75 and $0.45 \mu \mathrm{m}$ for $\mathrm{D}$.

simulated ones. We consider that the smaller decrease of light at the experimental PBG was caused by the variation of the diameter and the diffraction of light toward vertical directions.

\section{SPONTANEOUS LIFETIME AND INTERNAL EFFICIENCY}

The measurement setup of the phase-resolved spectroscopy is shown in Fig. 4. All the measurements were also carried out at room temperature. The CW pump light of $\lambda=1064$ $\mathrm{nm}$ was sinusoidally modulated with an angular frequency $\omega$ and focused on a sample from the top by an objective lens with a numerical aperture of 0.4 . The focused spot diameter of the pump light was $\sim 10 \mu \mathrm{m}$, and the irradiated power density was maximally $15 \mathrm{~kW} / \mathrm{cm}^{2}$. The absorbed power by the QW's was much lower than this value because of the thin QW's, i.e., 30 $\mathrm{nm}$ in total thickness. The PL radiated from columns toward upper direction was collimated by the same objective lens, transmitted through a bandpass filter of $\lambda=1.5-1.6 \mu \mathrm{m}$ and detected by a GaInAs pin photodiode. The pump light reflected at the sample surface was separately detected by the same photodiode. The phase shift between the two waves $\theta$ was repeatedly measured for various $\omega$ from 1 to $50 \mathrm{MHz}$. When the exponential decay is assumed as an impulse response of the relaxation process, the relation $\theta=\tan ^{-1}(\omega \tau)$ is satisfied for each $\omega$. A spontaneous lifetime $\tau$ is determined by the method of least squares for measured phase shifts and the relation. When the lifetime is dominated by the binary recombination, it is dependent on the pump density, so the phase shift should be dynamically changed for a deep modulation of the

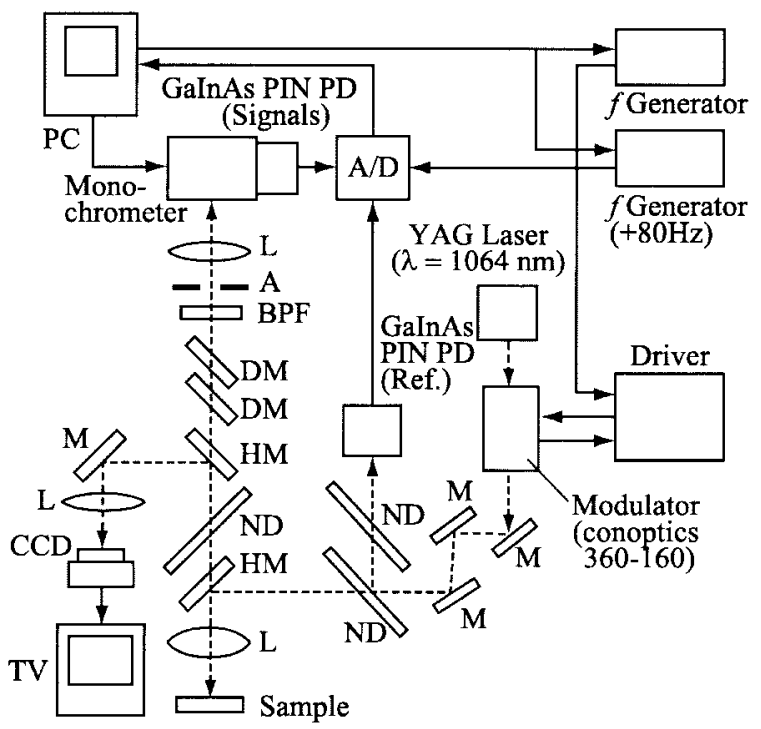

Fig. 4. Measurement setup of phase-resolved spectroscopy. Solid and dashed lines denote electrical and optical passes, respectively, and symbol M: mirror, HM: half mirror, L: lens, A: aperture, ND: neutral density filter, BPF: bandpass filter, and DM: dichroic mirror. Frequency of pump light and PL was reduced by making beat with $+80-\mathrm{Hz}$ electrical signal. Signal phase was determined against reference phase of pump light.

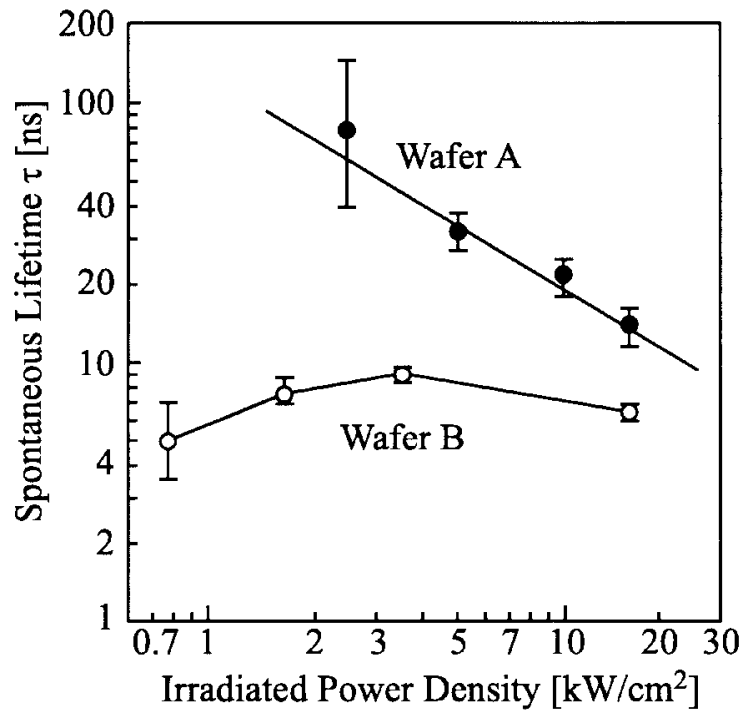

Fig. 5. Dependence of spontaneous lifetime of wafers A and B on irradiated power density of pump light.

pump light. In this experiment, the modulation depth was suppressed to less than $20 \%$ of the dc component to avoid this problem. As this method uses the CW pump, the PL from GaInAsP-InP columns could easily be detected with a high time-averaged power. Thus, this method is more advantageous than a time-resolved spectroscopy for which a low-noise and high-sensitivity detection is not available at $\lambda>1.1 \mu \mathrm{m}$.

Using this method, we first observed different characteristics of two as-grown wafers A and B. Fig. 5 shows the dependence of lifetime on the irradiated power density. For wafer A, the lifetime is inversely proportional to the power density. This is the typical dependence of the binary recombination. In contrast, the lifetime for wafer B was roughly independent 
of the power density and accordingly the carrier density in QW's. This was due to the doping against wafer B, in which the lifetime was dominated by the recombination with dopants against this pump level.

To evaluate the lifetime characteristic for columns in wafer $\mathrm{B}$, the nonradiative recombination at sidewalls of the active layer exposed to the air must be taken into account. In general, the inverse lifetime $\tau^{-1}$ is given by $\tau^{-1}=\tau_{r}^{-1}+\tau_{\mathrm{nr}}^{-1}$, where $\tau_{r}$ and $\tau_{\mathbf{n r}}$ are radiative and nonradiative recombination lifetimes, respectively. Let us ignore the nonradiative recombination at defects and interfaces inside the wafer. Let us also ignore the Auger recombination, since the absorbed power by the QW's was relatively small. Then, $\tau_{r}^{-1}=\gamma \tau_{\text {waf }}^{-1}$, where $\tau_{\text {waf }}$ is the wafer lifetime and $\gamma$ is the modulation factor of the radiative recombination by the $\mathrm{PBG}$, and $\tau_{\mathrm{nr}}^{-1}=2 v r^{-1}$, where $v$ is the nonradiative recombination velocity. When $\tau$ is changed by the surface recombination against a constant pump level, the carrier density in QW's should also be changed. Even under this condition, the lifetime for wafer B was roughly constant, as shown above. Therefore, $\tau$ is only dependent on $\gamma$ and $v r^{-1}$. Fig. 6 shows the dependence of the lifetime on the inverse radius, which was evaluated for columns on wafer B with various filling factor $f=0.12-0.17$. Each circle and error bar indicates, respectively, the average and the dispersion of lifetimes, which were repeatedly measured for each sample. The radius of columns in each sample was measured from SEM views. In Fig. 4, the inverse lifetime is almost proportional to the inverse radius with a slope of $3.2 \times 10^{4} \mathrm{~cm} / \mathrm{s}$. The velocity $v$ is $1.6 \times 10^{4} \mathrm{~cm} / \mathrm{s}$. After the wet etching of columns using $\mathrm{HCl}: \mathrm{H}_{2} \mathrm{O}=4: 1$ solution for $5 \mathrm{~s}$ at $2{ }^{\circ} \mathrm{C}, v$ was decreased to $80 \%$ of that before. Thus, the surface recombination velocity is $1.3 \times 10^{4} \mathrm{~cm} / \mathrm{s}$. This value is typical for GaInAsP-InP. The balance $0.3 \times 10^{4} \mathrm{~cm} / \mathrm{s}$ was caused by the etching damage and contamination. This result indicated that the internal efficiency $\eta_{\text {in }}=\tau / \tau_{\text {waf }}=$ $\left(1+2 v \tau_{\text {waf }} \gamma^{-1} r^{-1}\right)^{-1}$ was degraded by the nonradiative recombination.

In Fig. 6, data obtained for different filling factor $f$ are equally plotted, since the dependence on $f$ was not detected. In addition, the change of $\gamma$ was not detected even under the optimum condition for the PBG, i.e., $r^{-1} \sim 5 \mu \mathrm{m}^{-1}$ and $f=0.12$. For the honeycomb arrangement of columns, the width of the polarization insensitive PBG was calculated to be $\sim 10 \%$ of the center frequency $\omega$ [16]. Therefore, when the emission peak is tuned to $\omega$, the solid angle allowed for emission is restricted to $\sim 70 \%$ of the total solid angle $4 \pi$. We can expect $\sim 30 \%$ decrease of the inverse lifetime. In this experiment, however, the internal efficiency was $7 \%$ around the PBG condition. To evaluate the increase of the lifetime under this condition, $\sim 2 \%$ change of the lifetime must be detected. This seems to be difficult for the scattered data, as seen in Fig. 6. The change will not be detected without improving the internal efficiency to higher than $50 \%$.

\section{Light EXTRACTION EFFICIENCY}

The detected power $P_{\text {det }}$ of the PL was measured for each sample using the same setup, as shown in Fig. 4. Fig. 7 shows

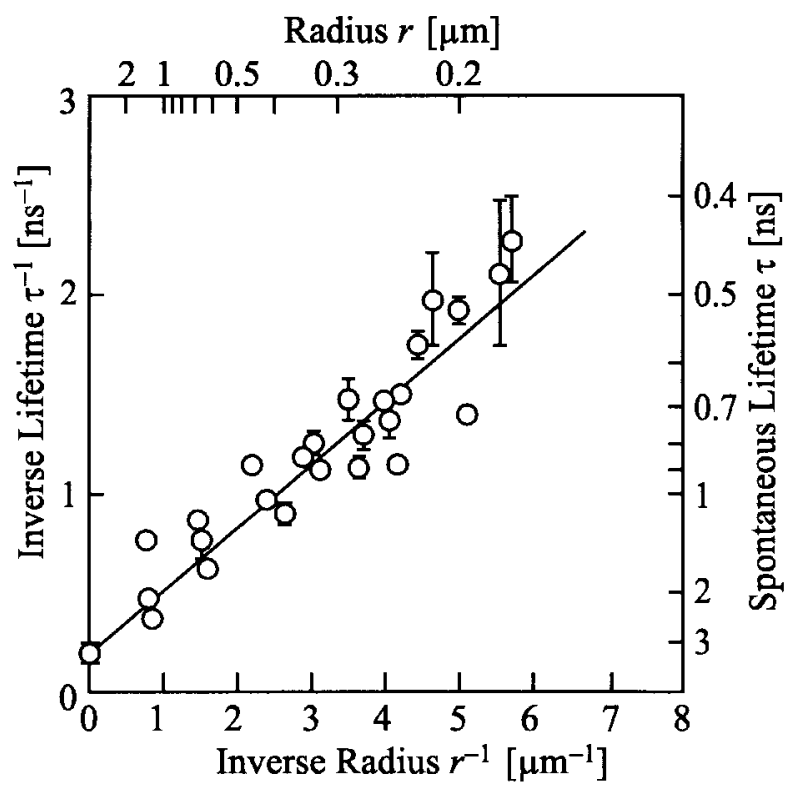

Fig. 6. Inverse lifetime for columns measured with inverse radius.

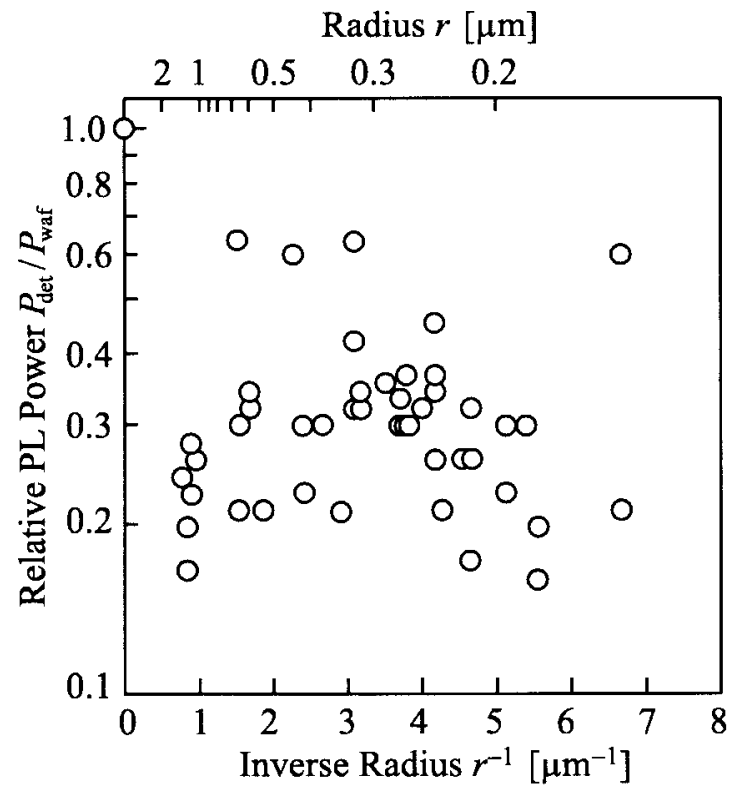

Fig. 7. Relative PL power measured with inverse radius.

the relative PL power $P_{\text {det }} / P_{\text {waf }}$ for all samples, where $P_{\text {waf }}$ is a typical PL power from as-grown wafer B. Data are scattered so that they give no significant properties without considering the filling factor $f$ and the internal efficiency $\eta_{\text {in }}$.

The extraction efficiency $\eta_{\mathrm{ex}}$ is expressed as $\eta_{\mathrm{ex}}=$ $P_{\text {det }} /\left(P_{\text {pump }} f \eta_{\text {abs }} \eta_{\text {in }} \eta_{\text {lens }}\right)$, where $P_{\text {pump }}$ is the pump power and $\eta_{\mathrm{abs}}$ and $\eta_{\text {lens }}$ are the absorption efficiency of the pump light and the collection efficiency of the PL by the objective lens, respectively. In this measurement, $P_{\text {pump }}$ and $\eta_{\text {ens }}$ were constant. First, let us assume $\eta_{\text {abs }}$ to be constant for simplicity. Using $P_{\text {det }}$ for the as-grown wafer B as a standard and taking into account the so-obtained $f$ and $\eta_{\text {in }}$ for each crystal, the enhancement of the light extraction efficiency was evaluated, as indicated by open circles in Fig. 8. A large enhancement over ten times was estimated 


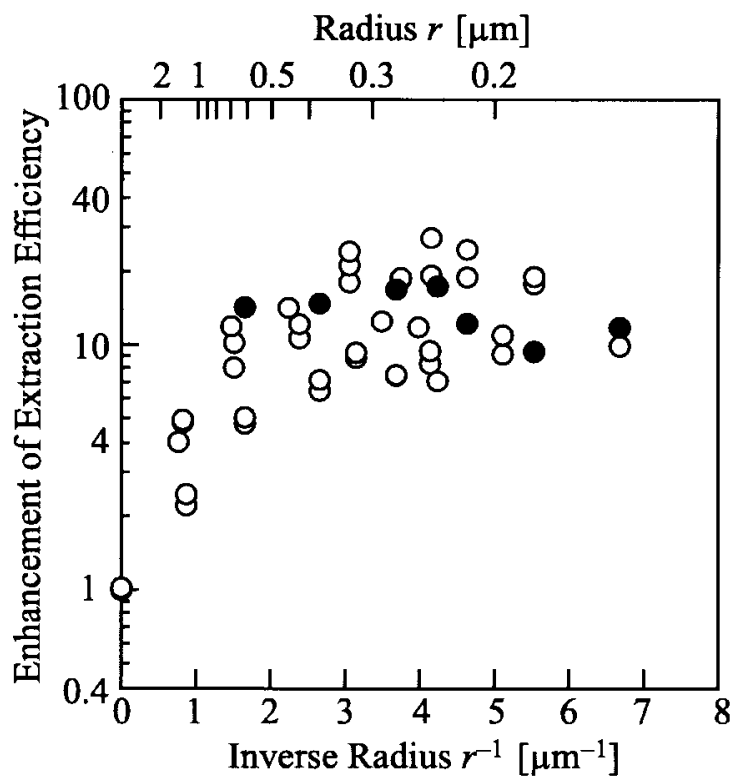

Fig. 8. Enhancement of light extraction efficiency evaluated for inverse radius. Open and closed circles denote those evaluated for pump light of $\lambda=1064$ and $840 \mathrm{~nm}$, respectively.

for smaller columns. The scattering of the data was thought to be due to the variation of the absorption efficiency $\eta_{\text {abs }}$. One may consider that the absorption efficiency is strongly changed with the change of the extraction efficiency. This is not true in this case, because the extraction efficiency is mainly restricted by the total reflection of the internal light, while the pump light can enter the structure without suffering the total reflection. We rather estimated that the absorption efficiency was of the same order for all samples, since drastic changes of the peak wavelength and the spectral shape of the PL were not observed. However, there still remain some possibilities of the small change of the absorption efficiency by the multireflection and repeated absorption in columns. To confirm the large enhancement of $\eta_{\mathrm{ex}}$, those for some samples were evaluated again using the pump light of $\lambda=840 \mathrm{~nm}$. For this wavelength, the InP claddings and substrate are strong absorbers, so the repeated absorption is suppressed. As indicated by closed circles in Fig. 8, the enhancement under this condition was still as large as 9-17 times. These values are almost the same as average values of those denoted by open circles. The extraction efficiency for a planar wafer is typically $2.4 \%$. Therefore, the enhancement corresponds to a high extraction efficiency of $22-41 \%$.

We considered the origin of the high efficiency. The extraction efficiency dominated by the escape cone of internal light is expressed as

$$
\eta_{\mathrm{ex}}=\left(1-\sqrt{1-\left(n_{0} / n\right)^{2}}\right) / 2
$$

where $n_{0}$ and $n$ are indexes of the air and the semiconductor, respectively. Let us apply this formula to the columns by considering the effective refractive index of the diluted semiconductor. It is generally difficult to determine uniquely the effective index of arranged columns due to the strong frequency dependence. Let us assume, for example, $n_{0}=1$ and $n=1.3$. Then, $\eta_{\mathrm{ex}}$ is calculated to be $18 \%$. It is still much lower than the highest value obtained in the experiment. The difference comes from the lack of considerations for the light toward lateral directions. The lateral light is not extracted from a simple diluted semiconductor, while it is extracted from the microcolumns. In another study concerning a laser diode with a deep grating DBR, we carried out a finite difference time-domain simulation of light in a periodic structure of semiconductor vertical walls and air space, which has a similar side view to that of the formed columns [21]. We observed that the transmission of the lateral light through the grating was dependent on the design, e.g., the first- and the second-order grating conditions. However, the characteristic of light toward vertical directions was not sensitive to the design. The far-field pattern was strongly oriented toward vertical directions for any pitches and semiconductor widths; no striking changes were observed even under the secondorder grating condition. The ratio of up- and down-oriented light was roughly $4: 6$. These results suggest that the lateral light is diffracted in the air space and easily converted to the vertical light by the strong scattering at semiconductorair interfaces. It can also be explained as the second-order Bragg diffraction with a very large coupling coefficient of the grating. Almost the same situation can be considered for the formed columns. We consider that this is the reason why the extraction efficiency evaluated was higher than that explained by the diluted semiconductor and why the efficiency was not sensitive to the small change of the radius.

\section{CONCLUSION}

GaInAsP-InP 2-D-arranged microcolumns with a high aspect ratio over eight were fabricated by the ICP etching. Using the direct optical pump of columns and the detection of the inplane PL, the spectral shift of the PBG was observed with the light suppression of $2-3 \mathrm{~dB}$. The scattering matrix simulation explained that this small decrease was mainly affected by the direct detection of light from edge columns. The lifetime measurement using the phase-resolved spectroscopy showed that the internal efficiency was reduced by the typical surface recombination of $1.3 \times 10^{4} \mathrm{~cm} / \mathrm{s}$ in velocity for this material system. Taking account of the internal efficiency and the filling factor of columns, a large enhancement of the extraction efficiency over ten times that for a planar wafer was evaluated.

This high efficiency was not primarily caused by the PBG but by the expanded escape cone in the diluted semiconductor and by the strong diffraction and scattering of lateral light. These effects were not sensitive to the structural design. Therefore, they are also expected for disordered columns fabricated by some easier process such as an anodic etching [22]. However, these effects are essentially different from the scattering at textured surface. The diluted semiconductor effect requires built-in active layers in columns. The diffraction and scattering discussed here cannot be obtained by textured surfaces. They are rather similar to those expected for small particle emitters.

The important issue to be investigated is the reduction of the surface recombination. The chemical treatment after the dry etching and the surrounding insulator should be optimized 
to reduce the surface recombination. For this purpose, another interesting challenge is the usage of other materials exhibiting small nonradiative recombination, e.g., GaN.

\section{ACKNOWLEDGMENT}

The authors would like to thank Prof. Y. Kokubun, Yokohama National University, for encouragement and valuable discussion.

\section{REFERENCES}

[1] T. Baba, "Photonic crystals and microdisk cavities based on GaInAsP/InP system," IEEE J. Quantum Electron., vol. 3, pp. 808-830, 1997.

[2] E. Yablonovitch, T. J. Gmitter, and R. Bhat, "Inhibited and enhanced spontaneous emission from optically thin $\mathrm{AlGaAs} / \mathrm{GaAs}$ double heterostructures," Phys. Rev. Lett., vol. 61, pp. 2546-2549, 1988.

[3] E. M. Purcell, "Spontaneous emission probabilities at radio frequencies," Phys. Rev., vol. 69, p. 681, 1946.

[4] T. Kobayashi, T. Segawa, Y. Morimoto, and T. Sueta, "Novel-type lasers, emitting devices, and functional optical devices by controlling spontaneous emission," in Proc. 46th Fall Meeting Japan Soc. Appl. Phys., 1982, no. 29a-B-6 (in Japanese).

[5] E. Yablonovitch, "Inhibited spontaneous emission in solid-state physics and electronics," Phys. Rev. Lett., vol. 58, pp. 2059-2060, 1987.

[6] Y. Yamamoto, S. Machida, K. Igeta, and G. Björk, "Controlled spontaneous emission in microcavity semiconductor lasers," in Coherence, Amplification and Quantum Effects in Semiconductor Lasers, Y. Yamamoto, Ed. New York: Wiley, 1991.

[7] T. Baba, T. Hamano, F. Koyama, and K. Iga, "Spontaneous emission factor of a microcavity DBR surface emitting laser," IEEE J. Quantum Electron., vol. 27, pp. 1347-1358, 1991.

[8] T. Nishizawa, T. Kakimura, Y. Lee, and M. Yamanishi, "Enhanced transfer efficiency in AlGaAs asymmetric planar microcavity light emitting diodes," Appl. Phys. Lett., vol. 65, pp. 1796-1798, 1994.

[9] H. Benisty, H. De Neve, and C. Weisbuch, "Impact of planar microcavity effects of light extraction-Part I: Basic concepts and analytical trends," IEEE J. Quantum Electron., vol. 34, pp. 1612-1631, 1998.

[10] J. Blondelle, H. De Neve, G. Borghs, P. Bandaele, P. Demester, and R. Baets, "High efficiency (>20\%) microcavity LED's," in Proc. Inst. Elect. Eng. Colloquium Semiconductor Optical Microcavity Devices and Photonic Bandgaps, London, U.K., 1996.

[11] S. Fan, P. R. Villeneuve, and J. D. Joannopoulos, "High extraction efficiency of spontaneous emission from slabs of photonic crystals," Phys. Rev. Lett., vol. 78, pp. 3294-3297, 1997.

[12] F. Stern and J. M. Woodall, "Photon recycling in semiconductor lasers," J. Appl. Phys., vol. 45, pp. 3904-3906, 1974.

[13] I. Schnitzer, E. Yablonovitch, C. Caneau, and T. J. Gmitter, "Ultrahigh spontaneous emission quantum efficiency, $99.7 \%$ internally and $72 \%$ externally, from AlGaAs/GaAs/AlGaAs double heterostructures," Appl. Phys. Lett., vol. 62, pp. 131-133, 1993.

[14] T. Baba, R. Watanabe, K. Asano, F. Koyama, and K. Iga, "Theoretical and experimental estimations of photon recycling effect in light emitting devices with a metal mirror," Japan J. Appl. Phys., vol. 35, pp. 97-100, 1996.

[15] I. Schnitzer, E. Yablonovitch, C. Caneau, T. J. Gmitter, and A. Scherer, "30\% external quantum efficiency from surface textured, thin-film lightemitting diodes," Appl. Phys. Lett., vol. 63, pp. 2174-2176, 1993.

[16] J. D. Joannopoulos, R. D. Meade, and J. N. Winn, Photonic Crystals-Molding The Flow of Light. Princeton, NJ: Princeton University Press, 1995

[17] A. Matsutani, F. Koyama, and K. Iga, "Low bias voltage dry etching of $\mathrm{InP}$ by inductively coupled plasma using $\mathrm{SiCl}_{4} / \mathrm{Ar}$," Jpn. J. Appl. Phys., vol. 37, pp. 6655-6656, 1999.

[18] J. Yonekura, M. Ikeda, and T. Baba, "Analysis of lightwave propagation in finite 2-D photonic crystals and related photonic circuits using the scattering matrix method," J. Lightwave Technol., vol. 17, pp. $1500-1508,1999$

[19] D. Labilloy, H. Benisty, C. Weisbuch, T. F. Krauss, R. Houdre, and U. Oesterle, "Use of guided spontaneous emission of a semiconductor to probe the optical properties of two-dimensional photonic crystals," Appl. Phys. Lett., vol. 71, pp. 738-740, 1997.

[20] T. Baba, M. Ikeda, N. Kamizawa, and H. G. Blom, "Photonic crystals and their applications," Ouyobutsuri (Appl. Phys.), vol. 67, no. 9, pp. 1041-1045, 1998 (in Japanese).
[21] M. Ariga, Y. Sekido, T. Baba, A. Matsutani, F. Koyama, and K. Iga, "Low threshold GaInAsP lasers with semiconductor/air DBR fabricated by inductively coupled plasma etching," in Proc. 3rd Conf. Laser Electro-Optics Pacific Rim, Seoul, Korea, 1999, no. FU6.

[22] T. Baba and M. Koma, "Possibility of InP-based 2-dimensional photonic crystal-An approach by anodization technique," Japan J. Appl. Phys., vol. 34, no. 2B, pp. 1405-1408, 1995.

Toshihiko Baba (M'93) was born in Nagano Prefecture, Japan, on November 12, 1962. He received the B.E., M.E., and Dr.E. degrees from the Division of Electrical and Computer Engineering, Yokohama National University, Yokohama, Japan, in 1985, 1987, and 1990, respectively.

He was engaged in antiresonant reflecting optical waveguides (ARROW's) and integrated lightwave circuits. In 1990, he joined the Precision and Intelligence Laboratory, Tokyo Institute of Technology, as a Research Associate and started research on vertical cavity surface emitting lasers (VCSEL's). In 1991, he reported the first calculation of spontaneous emission factor in VCSEL's. In 1993, he achieved the first RT CW operation of long-wavelength VCSEL. In 1994, he became an Associate Professor at Yokohama National University. He was a Guest Associate Professor at the Tokyo Institute of Technology from 1994 to 1998. His current interests are microcavity lasers, photonic band crystals, spontaneous emission control, micromechanical optics, nonlinear optics, and optical computing.

Dr. Baba is a member of the Institute of Electronics, Information and Communication Engineers (IEICE) of Japan, the Japan Society of Applied Physics, and the American Institute of Physics. He received the Niwa Memorial Prize in 1991, the Best Paper Award from the Micro-optic Conference in 1993 and 1999, and the Paper Award and Academic Encouragement Award from the IEICE in 1994.

Kyoji Inoshita was born in Kagawa Prefecture, Japan, on June 11, 1974 He received the B.E. degree from the Division of Electrical and Computer Engineering, Yokohama National University, Yokohama, Japan, in 1999 where he is currently pursuing the master's degree.

His thesis topic is the fine dry etching process using an inductively coupled plasma etching and a light emitter based on photonic bandgap structures.

Mr. Inoshita is a member of the Japan Society of Applied Physics.

Hiroko Tanaka was born in Yamaguchi Prefecture, Japan, on November 8, 1976. She received the B.E. degree from the Division of Electrical and Computer Engineering, Yokohama National University, Yokohama, Japan, in 1999, respectively

She currently is with NEC Corp. During her thesis work, she studied the phase-resolved spectroscopy of semiconductor microstructures.

Jun Yonekura was born in Shizuoka Prefecture, Japan, on July 19, 1973. He received the B.E. and M.E. degrees from the Division of Electrical and Computer Engineering, Yokohama National University, Yokohama, Japan, in 1997 and 1999, respectively.

$\mathrm{He}$ is currently with Canon, Inc. During his thesis work, he studied the scattering matrix analysis and fabrication of photonic crystal lightwave circuits.

Mr. Yonekura is a member of the Japan Society of Applied Physics.

Maiko Ariga was born in Nara, Japan, on August 31, 1974. She received the B.E. degree from the Division of Electrical and Computer Engineering, Yokohama National University, Yokohama, Japan, in 1998, where she currently is pursuing the master's degree.

Her thesis theme is the FDTD simulation and microfabrication of short cavity semiconductor lasers with semiconductor and air deep grating DBR's.

Ms. Ariga is a member of the Japan Society of Applied Physics. 
Akihiro Matsutani was born in Tokyo, Japan, on May 7, 1964. He received the B.S. degree in applied physics from the National Defense Academy, Yokosuka, Japan, in 1989 and the Dr.E. degree from the Tokyo Institute of Technology in 1999.

In 1989, he joined the Precision and Intelligence Laboratory, Tokyo Institute of Technology, as a Technical Officer. He is performing research on semiconductor microprocessing.

Dr. Matsutani is a member of the Japan Society of Applied Physics.

Tomoyuki Miyamoto was born in Tokyo, Japan, in 1968. He received the B.E., M.E., and Ph.D. degrees from the Tokyo Institute of Technology in 1968, 1991, and 1993, respectively.

During his Ph.D. work, he developed a $1.55-\mu \mathrm{m}$ wavelength vertical cavity surface emitting laser using chemical beam epitaxy. In 1996, he joined the Precision and Intelligence Laboratory, Tokyo Institute of Technology, as a Research Associate. In 1998, he became a Lecturer with the Research Center for Quantum Effect Electronics, Tokyo Institute of Technology. He is presently engaged in the research on vertical cavity surface emitting lasers, crystal growth of III-V semiconductors, and semiconductor quantum structures.

Dr. Miyamoto is a member of the Japan Society of Applied Physics, and the Institute of Electronics, Information and Communication Engineers (IEICE) of Japan.

Fumio Koyama (M'85) was born in Tokyo, Japan, on May 16, 1957. He received the B.S., M.S., and Ph.D. degrees in physical electronics from the Tokyo Institute of Technology in 1980, 1982, and 1985, respectively.

During his Ph.D. work, he studied dynamic single-mode lasers. In 1985, he joined the Precision and Intelligence Laboratory, Tokyo Institute of Technology, as a Research Associate. In 1988, he became an Associate Professor. Currently, he is doing research on photonic integrated WDM devices, surface emitting lasers, and related semiconductor microfabrication technologies. From 1992 to 1993, he was with AT\&T Bell Labs, Crawford Hill, NJ, as a Visiting Member of Technical Staff.

Dr. Koyama received the IEEE Student Paper Award in 1985, the Institute of Electrical Engineers Electronics Letters Premium in 1985 and 1988, the Paper Award and Shinohara Memorial Award from the Institute of Electronics, Information and Communication Engineers (IEICE) of Japan in 1990, and the Marubun Scientific Award from the Marubun Research Promotion Foundation in 1998. He is a member of the Japan Society of Applied Physics and the IEICE.
Kenichi Iga (F'88) was born in Hiroshima Prefecture, Japan, in 1940. He received the B.E., M.E., and Dr.Eng. degrees from the Tokyo Institute of Technology, Tokyo, Japan, in 1963, 1965, and 1968, respectively.

In 1968, he joined the Precision and Intelligence Laboratory, Tokyo Institute of Technology, and became Associate Professor in 1973 and Professor in 1984. $\mathrm{He}$ is also responsible for education in the Interdisciplinary Graduate School. He has held the Teiichi Yamazaki Chair since 1993. He was Director of the Precision and Intelligence Laboratory from 1995 to 1998 . He is presently engaged in research on surface-emitting semiconductor lasers and microoptic systems, mostly on 2-D arrayed devices. From 1979 to 1980, he was with Bell Laboratories, Holmdel, NJ, as a Visiting Technical Staff Member and was engaged in GaInAsP/InP technology for single-mode lasers and integrated optics. He first proposed and pioneered the research of surface emitting semiconductor lasers. His idea has in recent years triggered much research in surface emitting lasers, which have become very important as coherent light-sources with 2-D parallelism. He is an active proponent of micro-optics, utilizing gradient-index microlens array, and is working toward realizing 2-D arrayed optical devices in combination with surface emitting lasers. Recently, his proposed multiquantum barrier has been introduced into visible semiconductor lasers.

Dr. Iga is a Fellow of the Optical Society of America (OSA) and a member of the Institute of Electronics, Information and Communication Engineers of Japan (IEICE). He is a member of the Board of Governors of the IEEE Lasers and Electro-Optics Society. He was a Japanese Subcommittee Chair of CLEO during 1988-1990. He has been a member of the program committees of all Topical Meetings on Gradient-Index Optical Imaging Systems since 1979 and was a Conference Chair of its 1989 Meeting. At the time of OSA's 70-year anniversary, he was chosen as one of 70 distinguished optical people. $\mathrm{He}$ was Chair of the Opto-Quantum Electronics Group in 1990. He is one of the Elected Members of Editors for 1993 and 1994. He was President of the IEICE Electronics Society in 1996. In the Optical Society of Japan and the Japan Society of Applied Physics, he organized the Microoptic Group, which he has chaired since 1988 